ing the dose of bronchodilator drug which reaches the bronchi. This problem was not of course resolved by the use of IPPV, which merely ensured that about the same volume of aerosol was delivered to the bronchi whatever drug or concentration was used. It is probable, however, that the average dose of isoprenaline received by the patients in this particular study was relatively large, because the increase in heart rate (29 per minute) produced by a $0.5 \%$ concentration given for three minutes by IPPV was much greater than that produced by $1,000 \mu \mathrm{g}$. of isoprenaline administered by pressurized dispenser (13 per minute) in a previous study reported by Choo-Kang et al. (1969) on a similar group of patients. The average dose of salbutamol received by the patients must have been equally large when a $0.5 \%$ concentration was used, but it caused a much smaller increase in heart rate.

IPPV has the capability, which a pressurized dispenser lacks, of delivering an adequate dose of bronchodilator aerosol to dyspnoeic patients. Such patients are often unable to inhale more than a small proportion of a single dose of aerosol from a pressurized dispenser, and this may partly account for the poor response of patients with status asthmaticus to bronchodilator drugs administered in that way. Furthermore, when the technique of IPPV is used, the drug is administered in a high concentration ( $40 \%$ or more) of oxygen, and if the studies in experimental animals with isoprenaline reported by Collins et al. (1969) are applicable to man this may reduce considerably the potential cardiotoxic hazards associated with the use of bronchodilator aerosols.

Though it cannot, of course, be assumed that a concentration of salbutamol which does not produce tachycardia is ipso facto less likely to provoke more serious cardiac side effects, it would seem reasonable, when powerful bronchodilatation is required, to select a form of treatment which does not increase the heart rate, and which at the same time ensures adequate oxygenation. These requirements can substantially be met by the administration of a $0.5 \%$ aerosol of salbutamol by IPPV in $40 \%$ oxygen for a period of three minutes.

The solutions of salbutamol and isoprenaline were supplied by Allen \& Hanburys Limited. We are indebted to Dr. D. Jack and Dr. W. T. Simpson for advice on the design of the trial, to Mrs. Barbara Dow for technical services, and to Mr. A. J. Davey for the statistical analysis.

REFERENCES

Choo-Kang, Y. F. J., Simpson, W. T., and Grant, I. W. B. (1969). British Medical fournal, 2, 287

Collins, J. M., McDevitt, D. G., Shanks, R. G., and Swanton, J. G. (1969) British fournal of Pharmacology, 36, 35

Hartley, D., Jack, D., Lunts, L. H. S., and Ritchie, A. C. (1968). Nature, 219, 861 .

Kennedy, M. C. S., and Simpson, W. T. (1969). British Fournal of Diseases of the Chest, 63, 165 .

Riding, W. D., Chatterjee, S. S., and Dinda, P. (1969). British Journal of Clinical Practice, 23, 217.

Warrell, D. A., et al. (1970). British Medical fournal, 1, 65.

\title{
Suppression of Ventricular Tachyarrhythmias by Transvenous Intracardiac Pacing after Acute Myocardial Infarction
}

\author{
M. A. BENNETT, ${ }^{*}$ M.R.C.P. ; B. L. PENTECOST, $\dagger$ M.D., M.R.C.P.
}

British Medical fournal, 1970, 4, 468-470

\begin{abstract}
ummary: Transvenous intracardiac pacing was carried $S$ out on nine patients with recurrent ventricular tachyarrhythmia after drug treatment had failed. In eight patients recurrent ventricular ectopic activity was suppressed by pacing at a rate above the sinus rate. With this technique the need for $D . C$. shock and repeated antiarrhythmic drugs can be avoided.
\end{abstract}

Recurrent ventricular tachycardia often terminating in fibrillation may be unavoidable after myocardial infarction in spite of prophylactic drug therapy. Such rhythm disturbances may be suppressed by rapid cardiac pacing, ${ }^{1-16}$ but this technique appears to have been little used after acute infarction. Pacing for recurrent ventricular tachyarrhythmia has been made relevant and feasible in many general hospitals with the widespread establishment of coronary care units, in which patients are closely supervised during the peak incidence of ventricular arrhythmias. Fluoroscopy facilities within the coronary care unit allow positioning of the transvenous pacing catheter without delay. This communication describes cardiac pacing in nine successive patients with recurrent ventricular tachyarrhythmia complicating acute myocardial infarction.

\section{Methods}

All nine patients were admitted to the hospital's coronary care unit. Before developing ventricular tachyarrhythmia they showed no evidence of cardiac failure, sinus bradycardia, or atrioventricular block; however, radiographic evidence of pulmonary oedema had developed in all nine at the time pacing was begun. The patients had not received a digitalis preparation and had normal serum electrolytes. Over 10 episodes of cardiac arrest were experienced by each patient (Table I). The

* Research Fellow.

t Consultant Physician.

Coronary Care Unit, General Hospital, Birmingham 4. term "cardiac arrest" is used here to indicate impairment of consciousness secondary to ventricular tachyarrhythmia which did not spontaneously revert to sinus rhythm.

After the initial episode of arrest the patients received prophylactic lignocaine by continuous infusion; additional chemotherapy was given if this failed to suppress ventricular ectopic activity (Table II). All episodes of prolonged ventricular tachycardia or ventricular fibrillation were terminated by D.C. shock. Pacing was started when drug therapy was considered to have failed; lack of definition of this failure point resulted in a variable delay before pacing. A transvenous bipolar catheter (6F U.S.C.I.) and a fixed-rate pacemaker (Medical Electronics and Instruments, Birmingham) were used. The catheter was introduced into a medial arm vein at

\begin{tabular}{|c|c|c|c|c|c|c|}
\hline Case No. & & & Sex & Age & Infarction Site & No. of Arrests \\
\hline $\begin{array}{l}1 \\
2 \\
3 \\
4 \\
5 \\
6 \\
7 \\
8 \\
9\end{array}$ & $\begin{array}{l}\ldots \\
\cdots \\
\cdots \\
\cdots \\
\cdots \\
\cdots\end{array}$ & $\begin{array}{l}\ldots \\
\cdots \\
\cdots \\
\cdots \\
\cdots \\
\cdots \\
\cdots\end{array}$ & $\begin{array}{l}\text { M. } \\
M . \\
M . \\
F . \\
F . \\
M . \\
F . \\
M . \\
M .\end{array}$ & $\begin{array}{l}55 \\
42 \\
51 \\
48 \\
62 \\
47 \\
52 \\
57 \\
64\end{array}$ & $\begin{array}{l}\text { Anterior } \\
\text { Anterior } \\
\text { Anterior } \\
\text { Inferior } \\
\text { Anterior } \\
\text { Anterior } \\
\text { Anterior } \\
\text { Inferior } \\
\text { Inferior }\end{array}$ & $\begin{array}{l}28 \\
29 \\
24 \\
18 \\
22 \\
11 \\
27 \\
14 \\
19\end{array}$ \\
\hline
\end{tabular}

TABLF II.-Drug Therapy and its Duration of Administration Prepacing

\begin{tabular}{|c|c|c|c|c|}
\hline Case No. & & Lignocaine (mg.) & Procainamide (mg.) & Quinidine (mg.) \\
\hline $\begin{array}{l}1 \\
2 \\
3 \\
4 \\
5 \\
6 \\
7 \\
8 \\
9\end{array}$ & \begin{tabular}{l|}
$\cdots$ \\
$\cdots$ \\
$\cdots$ \\
$\cdots$ \\
$\cdots$ \\
$\cdots$ \\
$\cdots$ \\
$\cdots$
\end{tabular} & 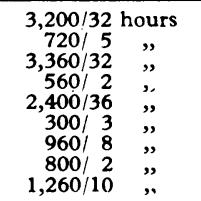 & 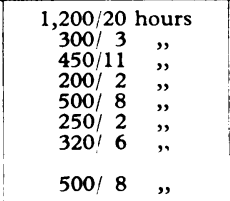 & $\begin{array}{l}500 / 8 \text { hours } \\
1,500 / 35,\end{array}$ \\
\hline
\end{tabular}


the antecubital fossa and guided to a stable position within the heart under fluoroscopic control. The fluoroscopic equipment (Philips BV $20 \mathrm{~S}$ ) was available in the coronary care unit. Continuous recording of the electrocardiogram on electromagnetic tape allowed an accurate assessment of the results of cardiac pacing. Permanent paper records from the tape were made with an electrocardiograph (Mingograph 24B) at a paper speed of $25 \mathrm{~mm}$./sec.

Atrial pacing at 5 volts was always attempted first. Ventricular pacing at 2 volts was used only if the pacing catheter in the right atrium was unstable or if atrial pacing failed to suppress ventricular irritability. In these circumstances ventricular pacing was started with a catheter positioned at the apex of the right ventricle. The initial artificial rate, which was always faster than the sinus rate, was gradually increased until ventricular ectopic activity disappeared.

\section{Results}

A stable position of the pacing catheter in the right atrium could be found only in Cases 1, 3, and 8. Ventricular pacing was therefore used in the other six patients. Suppression of ventricular ectopic activity was achieved in all nine patients (Figs. 1-3). In eight suppression occurred after an artificial increase in heart rate above the sinus rate (Table III). Case 8 , however, when paced at an atrial rate of 136 beats/min., continued to manifest ventricular ectopic activity. This was abolished by advancing the catheter to the ventricle and pacing at a similar rate to the pre-arrest sinus rate of 125 beats $/ \mathrm{min}$. Chemotherapy was stopped on the introduction of pacing. Case 7, however, during the second day of pacing also required lignocaine $1 \mathrm{mg} . / \mathrm{min}$. and procainamide 0.25 $\mathrm{mg}$./min. to suppress ventricular premature beats.

When pacing was stopped during the first 72 hours ventricular premature beats were noted in all nine patients. In addition Cases 5 and 6 had prolonged episodes of ventricular tachycardia, which were reverted to sinus rhythm by pacemaker stimulation. ${ }^{17}$ Cardiac pacing was stopped in Case 4 within 48 hours; the patient was then discharged to a general medical ward, where after 20 hours she developed ventricular fibrillation and died. The other patients were paced for five days.

The eight patients who survived the first 72 hours had no further episodes of ventricular tachyarrhythmia. All were discharged from hospital and were still alive at the time of writing, with varied survival times of 2 to 12 months.

\section{Discussion}

In eight patients suppression of recurrent ventricular ectopic activity was achieved by an increase in heart rate above the sinus rate. This fact may be explained by the results of some animal experiments. After myocardial infarction in the dog repolarization has been shown to be irregular; thus an ectopic focus may arise as a potential difference between adjacent polarized and depolarized tissue. If an ectopic focus initiated in such a way discharges prematurely an even greater temporal dispersion of repolarization will

TABLE III.-Ventricular Ectopic Activity Suppressed by Artificial Increase in Heart Rate

\begin{tabular}{cc|c|c}
\hline Case No. & & Sinus Rate Pre-arrest & $\begin{array}{c}\text { Paced Rate which Suppressed } \\
\text { Ectopic Activity }\end{array}$ \\
\hline 1 & $\ldots$ & 75 & 136 \\
2 & $\ldots$ & 89 & 125 \\
3 & $\cdots$ & 100 & 111 \\
4 & $\cdots$ & 725 & 129 \\
5 & $\cdots$ & 83 & 115 \\
6 & $\cdots$ & 75 & 91 \\
7 & $\cdots$ & 125 & 125 \\
8 & $\cdots$ & 75 & 110 \\
\hline
\end{tabular}

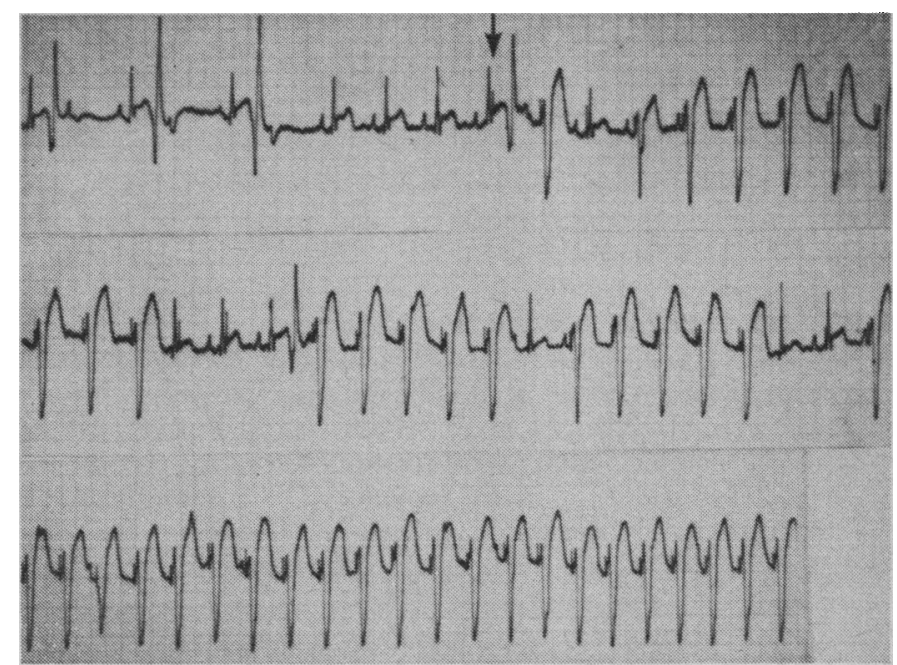

Fig. 1.-Top: Ventricular premature complexes displaying the $R / T$ phenomenon. Arrow indicates first pacemaker artefact. Middle and Bottom Ventricular capture and suppression of ectopic activity achieved by gradual increase in paced ventricular rate.

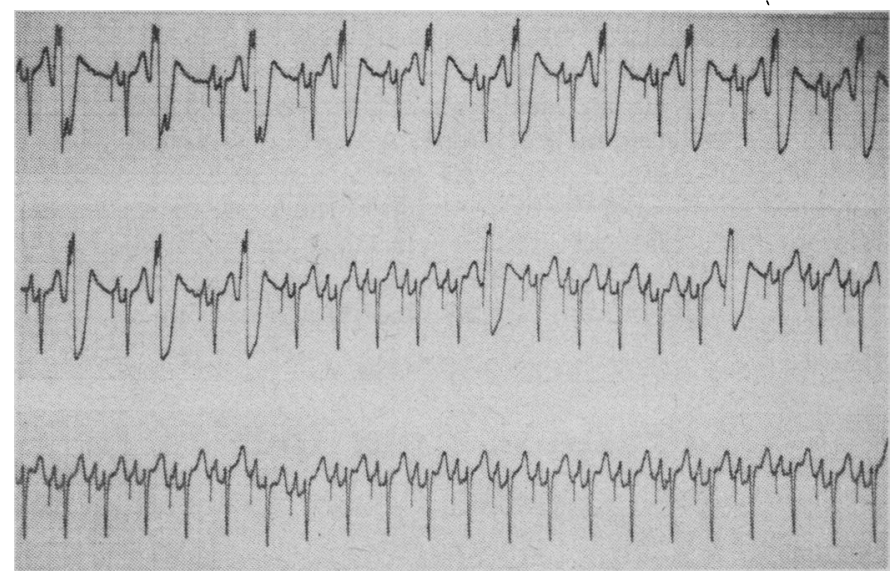
FIG. 2.- Top: Coupled premature extrasystoles despite atrial pacing.
Middle and Bottom: Gradual increase in rate results in suppression of ventricular ectopic activity.

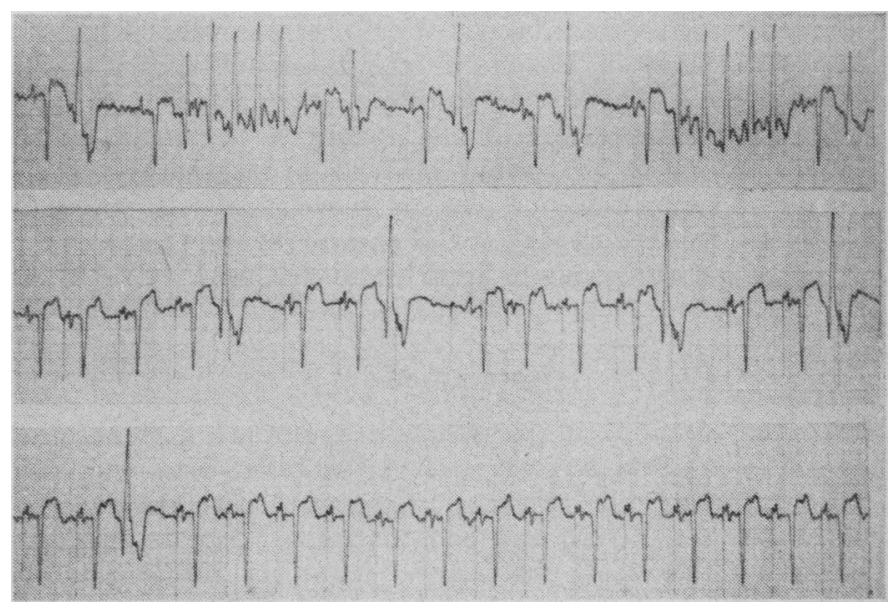

FIG. 3.-Top: Multifocal ventricular ectopic activity. Middle and Bottom: Gradual increase in paced atrial rate with ventricular ectopic suppression.

result, ${ }^{19}$ thereby increasing the possibility of multiple ectopic foci, whose combined asynchronous discharge may cause ventricular fibrillation. Irregular repolarization and premature ectopic activity of the ischaemic dog ventricle have been shown, however, to disappear with an artificial increase in heart rate. ${ }^{20}$ If myocardial infarction in man causes similar irregular repolarization which results in ventricular ectopic 
activity, then an artificial increase in heart rate may achieve its antiarrhythmic effect by creating regular repolarization.

Ventricular ectopic activity was avoided in one patient by ventricular pacing at a similar rate to the sinus rate after more rapid atrial pacing had failed. The generation of ventricular ectopic activity in this patient may have been related to the creation of decremental block and its resultant re-entry circuits in the ischaemic His-Purkinje tissue. ${ }^{21}$ An alteration in the impulse pathway may have avoided the initiation of such block and therefore its arrhythmic consequences.

The exact time to introduce this form of therapy must be arbitrary. More recently at this hospital all patients with two cardiac arrests within 24 hours or one arrest with continued ventricular ectopic activity were paced. The ventricle is the site of choice for pacing in these patients. The instability of the pacing catheter in the atrium makes atrial pacing over days impracticable. Because ventricular ectopic activity was noted on stopping pacing during the first 72 hours in all patients, continued pacing for at least five days is now performed. Permanent pacing, however, has not been required in any of the eight survivors.

We would not advise the universal use of this method because many episodes of ventricular tachycardia are not recurrent, and it would be unjustified to use a technique which involves cardiac catheterization and inevitably some risk to the patient. Nevertheless, given the right indications, transvenous cardiac pacing can reduce the need for antiarrhythmic drugs, which themselves can precipitate ventricular tachyarrhythmias and cardiac failure. ${ }^{22-26}$ In our patients it reduced the need for D.C. shock and therefore avoided the possible myocardial damage that may result after electrical cardioversion. ${ }^{27}$ The avoidance of arrest gives the patients confidence in recovery and lightens the burden of busy medical and nursing staff.
REFERENCES

1 Sowton, E., Leatham, A., and Carson, P., Lancet, 1964, 2, 1098.

Swedberg, J., and Malm, A., Acta Chirurgica Scandinavica, 1964, 128,

${ }^{3}$ Eraklis, A. J., Green, W. T., and Watson, C. G., Annals of Surgery, $1965,161,63$.

- Heiman, D., and Helwig, J., fournal of the American Medical Association, 1966, 195, 1150.

5 Schoonmaker, F. W., Osteen, R. T., and Greenfield, J. C., Annals of Internal Medicine, 1966, 65, 1076.

- McCallister, D., McGoon, D. C., and Connolly, D. C., American fournal

- Kastor, J. A., De Sanctis, R. W., Harthorne, J. W., and Schwartz, G.,

Annals of Internal Medicine, 1967, 66, 939. Annals of Internal Medicine, 1967, 66, 945

- Lewn, H. T., and March, H. W., American Heart fournal, 1967, 73, 794.

10 Tancredi, R. G., McCallister, B. D., and Mankin, H. T., Circulation, 1967, 36, 598 . 11 Zipes, D. P., and Orgain, E. S., Annals of Internal Medicine, 1967, 67,

12 I.oeb, H. S., Pietras, R. J., Gunnar, R. M., and Tobin, J., Circulation, 1968, 38, 210 .

13 Zipes, D. P., et al., Annals of Internal Medicine, 1968, 68, 591.

14 Moss, A. J., Rivers, R. J., Griffith, L. S., Carmel, J., and Millard, E., New England fournal of Medicine, 1968, 278, 928 .

15 Hornbaker, J. H., Humphries, J. O., and Ross, R. S., Circulation, 1969, $39,189$.

16 Friedberg, C. K., Lyon, L. J., and Donoso, E., American Heart fournal, $1970,79,44$.

17 Bennett, M. A., and Pentecost, B. L., in preparation.

18 Han, J., and Moe, G. K., Circulation Research, 1964, 14, 44

Han, J., Garcia de Jalon, P., and Moe, G. K., Circulation Research, $1966,18,18$.

so Han, J., De Tralgia, J., Millet, D., and Moe, G., American Heart fourna!, $1966,72,632$.

21 Hoffman, B. F., and Cranefield, P. F., American fournal of Medicine, 1964, 37, 670.

22 Kayden, H. J., Progress in Cardiovascular Diseases, 1961, 3, 331.

23 Castellanos, A., and Salhanick, L., American fournal of the Medical Sciences, 1967, 253, 52.

24 Davies, P., Leak, D., and Oram, S., British Medical fournal, 1965, 2, 517.

25 Kaufmann, G., Lancet, 1968, 1, 862.

26 Nagle, R. E., and Pilcher, J., Lancet, 1968, 1, 1039.

27 I.own, B., Amarasingham, R., and Neuman, J., fournal of the American Medical Association, 1962, 182, 548.

\title{
Prostatic Contribution to Normal Serum Acid Phosphatase
}

\author{
DONALD DOW, ${ }^{*}$ F.R.C.S.ED. ; ROBERT H. WHITAKER, ${ }^{*}$ M.CHIR., F.R.C.s.
}

\author{
British Medical fournal, 1970, 4, 470-472
}

\begin{abstract}
Cummary : Total and tartrate-labile serum acid phosphatase levels were compared in patients with and without prostates, and in 12 patients before and after cystoprostatectomy. Absence of the prostate seems to make no significant difference to the levels of serum acid phosphatase. There is no justification for referring to the tartrate-labile serum acid phosphatase as "prostatic acid phosphatase." A substantial incidence of marginally raised levels of serum acid phosphatase in each group of patients suggests that the upper limit of normal for the total serum acid phosphatase should be taken as 5 K.A.u.
\end{abstract}

\section{Introduction}

The total serum acid phosphatase comprises a mixture of phosphatases from most tissues of the body (Doe and Seal, 1965). Fishman and Lerner (1953) showed that the activity of part of the total serum acid phosphatase could be inhibited by L-tartrate. The acid phosphatase found in the prostate is tartrate-inhibitable (labile), as is also a large proportion of that found in the liver, spleen, and kidneys (Abul-Fadl and King, 1949).

It is not clear what contribution the various organs make to the total serum acid phosphatase and in particular to the tartrate-labile fraction. It has generally been assumed that the prostate is the major source of the normal serum tartratelabile acid phosphatase; hence this fraction has commonly

\footnotetext{
- Resident Surgical Officer, St. Peter's Hospital, London W.C.2.
}

been referred to as the "prostatic acid phosphatase." King and Jegatheesan (1959), however, noted that the mean values of the tartrate-labile acid phosphatase are practically the same for males and females, and concluded that this "prostatic" component in normal persons must come from sites other than the prostate. It was felt that a study of a series of patients undergoing total cystoprostatectomy might shed further light on the extent to which the prostate contributes to the normal serum acid phosphatase levels.

\section{Patients and Methods}

Total serum acid phosphatase and tartrate-labile acid phosphatase levels were determined in 100 patients. Of these, 25 were men who had total cystoprostatectomy for bladder cancer, and in 12 of these the determinations were made both before and after surgery. A detailed histological examination of the excised specimen showed that none of these patients had malignancy of the prostate. There were 25 men with no symptoms of prostatic disease, and a further 25 men who had estimations before transurethral or retropubic prostatectomy for what was subsequently shown histologically to be benign prostatic hypertrophy. Finally, determinations were made on 25 women-9 were healthy volunteers and 16 were women admitted for minor endoscopic procedures. There was no history, past or present, of carcinoma of the breast in any of these women.

Throughout, the King-Armstrong (1934) method as modified by Gutman and Gutman (1940) was used, and all results are expressed as King-Armstrong units per $100 \mathrm{ml}$. of 